\title{
Specific-heat anomaly near superconducting transition in high temperature superconductors
}

\author{
M P DAS \\ Department of Theoretical Physics, Research School of Physical Sciences, The Australian \\ National University, Canberra, ACT 2601, Australia
}

\begin{abstract}
A brief appraisal of the current state of understanding on the specific heat anomaly near $T_{c}$ in the oxide superconductors is presented. Spectacular specific heat double transitions are explained with plausible models.
\end{abstract}

Keywords. Specific heat; Ginzburg-Landau theory; order parameter; fluctuations; Gaussian approximation; double transitions.

\section{Introduction}

The specific heat anomaly near $T_{c}$ is a key signature on the nature of the superconducting phase transition. Now it is well-known that in the high $T_{c}$ superconductors (HTS) a complicated interplay of crystalline structure, chemical composition and method of preparation drastically affects the occurrence of superconductivity. Therefore, unlike in conventional superconductors, a clear observation of the specific heat transition(s) and its interpretation remained to be a controversial issue (for details see Choy et al 1989). Two recent reviews present the status reports regarding the specific heat studies (Fisher et al 1988; Fischer et al 1988). In this paper we discuss mainly the specific heat transition(s) around the critical temperature and point out its significance in the HTS.

\section{Why specific heat so important?}

A superconductor is conveniently characterized by one (or more) order parameter (OP)(s) possessing certain symmetries of the crystalline lattice, time reversal and the gauge group. In conventional superconductors only the gauge symmetry is broken, while all other symmetries are preserved. In the new (heavy electron and oxide) superconductors one or more of the other symmetries are also broken. Since the specific heat is a record of superconducting phase transition, a careful analysis ought to reveal the symmetry information, which would be relevant for understanding the pairing and hence the microscopic mechanism. If correctly analysed the specific heat data can possibly provide the number of order parameters (or number of components) and the spatial dimensions apart from quantities like density of states, Debye temperature etc.

\section{GL Theory}

In the absence of a clear microscopic picture, the Ginzburg-Landau (GL) theory provides a rigorous framework to understand the broken symmetries of the OP. The 
GL-free energy is expanded as a power series in the OP around $T_{c}$ and by using variational method the GL equations are set up. We note that the conventional GL theory invokes one complex $(n=2)$ OP. There can be various generalizations of the GL theory made by necessity for various physical situations (see Das 1989).

The solution of the GL equations depends on the approximations and the associated boundary conditions. The simplest one is the mean-field approximation (MFA). The free energy, $F$ is calculated in the MFA from which the specific heat, $C_{p}$ is obtained by $C_{p}=-T\left(\partial^{2} F / \partial T^{2}\right)$. MFA is adequate for conventional superconductors, because the critical region is too tiny to be experimentally accessible, i.e. $\Delta T_{c} \sim\left\{\kappa^{4} T_{c}^{3} / H_{c 2}(0)\right\} \times$ $10^{-9}$. Here $\kappa$ and $H_{c 2}(0)$ are the Gorkov parameter and the second critical field at $T=0$ respectively.

In HTS $\kappa$ is large and $T_{c}$ is also large, as a result the critical region is measurably wide $(\sim 0.5 \mathrm{~K})$. Therefore the fluctuations of the OP are important, which ought to be described beyond the MFA. Apart from specific heat, measurements of electrical resistivity, magnetic susceptibility and thermoelectric power have been reported showing fluctuation effects. There may be anisotropy but the fluctuations are threedimensional $\sim|\tau|^{-1 / 2}$, where $\tau=\left(T-T_{c}\right) / T_{c}$. To treat the fluctuations again, the simplest approximation is Gaussian. Using the method of Ma (1976), the electronic specific heat, $C(T)=C^{\mathrm{MF}}(T)+C^{\mathrm{Fl}}(T)$, where $\mathrm{MF}$ and $\mathrm{Fl}$ stand for mean field and fluctuation respectively. $C^{\mathrm{Fl}}$ is given by, $C^{\mathrm{Fl}}=C_{ \pm}|\tau|^{-2 \pm d / 2}$. Here $C_{+}$is the amplitude of the fluctuation contributions above and below $T_{c}$ and $d$ the spatial dimension. In the conventional GL theory the amplitude ratio $C_{+} / C_{-}=n / 2^{d / 2}$. Here $n$ is the number of components of the order parameter equal to 2 . Therefore $C_{+} / C_{-}=2^{-1 / 2}$ is a universal number for $d=3$.

If there is more than one transition the same procedure can be used to calculate the mean field and fluctuation contributions to the specific heat (Das et al 1988).

\section{Discussion}

Measurements of the specific heat exhibit jump(s) at the transition temperature, which are strongly sample-dependent. From a number of data (Fisher et al 1988; Fischer et al 1988) it is found that the magnitude of the jump $\left(\Delta C / \gamma T_{c}\right)$ is consistent with the predictions of the weak coupling BCS theory $(1.43)$ to within $30 \%$ in lanthanum and yttrium samples.

Fluctuation contributions to the specific heat were first reported by Inderhees et al (1988). These authors subtracted the mean field (BCS) contributions from their data and fitted $C_{+} / C_{-}$to the effective number of OP components lying in the range $5<n<10$. Though this procedure is not correct as has been pointed out by Muziker (1988), Das et al (1988) and many others, this work has been a subject of hot discussion (see Choy et al 1989). However, there is no consensus on the number and the nature of the order parameters in the analysis of data of Inderhees et al (1988). On the contrary, Ebner and Stroud (1988) calculated the specific heat via Monte-Carlo simulations and have concluded that the conventional one complex $(\mathrm{O}(2)) \mathrm{GL}$ theory can account for the shape of the specific heat data for $d=3$.

Experiments of Inderhees et al (1988) and many others (see for details Choy et al 1989) have observed well-resolved specific heat double transitions in (123) HTS with 
a temperature difference of $<5 \mathrm{~K}$. These results are analysed with two plausible models:

(i) twinning plane superconductivity (TPS) (Sobyanin and Stratonnikov 1988; Abrikosov et al 1988) and (ii) a two-order parameter GL theory (Das et al 1988). Important difference in these two methods lies in the fact that in the former localized superconductivity first occurs on the twinning plane and later the bulk becomes superconducting. In the latter a superconducting transition occurs again when the bulk is already superconducting. Both the approaches are based on the phenomenological GL formalism, therefore no microscopic explanation has been given.

We understand that there is a structural (tetragonal-orthorhombic) transition near $T_{c}$ (Horn et al 1987). Its occurrence depends on oxygen stoichiometry and preparation history. The high temperature normal phase is tetragonal and by lowering the temperature, the lattice becomes soft in the vicinity of the structural transition to favor a superconducting transition. If the structural transition temperature, $T_{0}$ does not coincide with $T_{c}$ but $T_{0}<T_{c}$, a second superconducting transition takes place. This transition is nearly first order as shown by Das et al (1988) in their two-order parameter GL theory. In figure 1, calculated specific heat within the Gaussian approximation is shown having two transitions in agreement with the data of Butera (1988). It is now possible to symmetry-classify the order parameters following Volovik and Gorkov (1985) (see also Volovik 1988). The associated symmetry of the OP is indicative of the nature of pairing.

Summarizing, we have presented a brief appraisal of the specific heat anomalies near $T_{c}$ in the HTS. Spectacular specific heat double transitions are explained with plausible models.

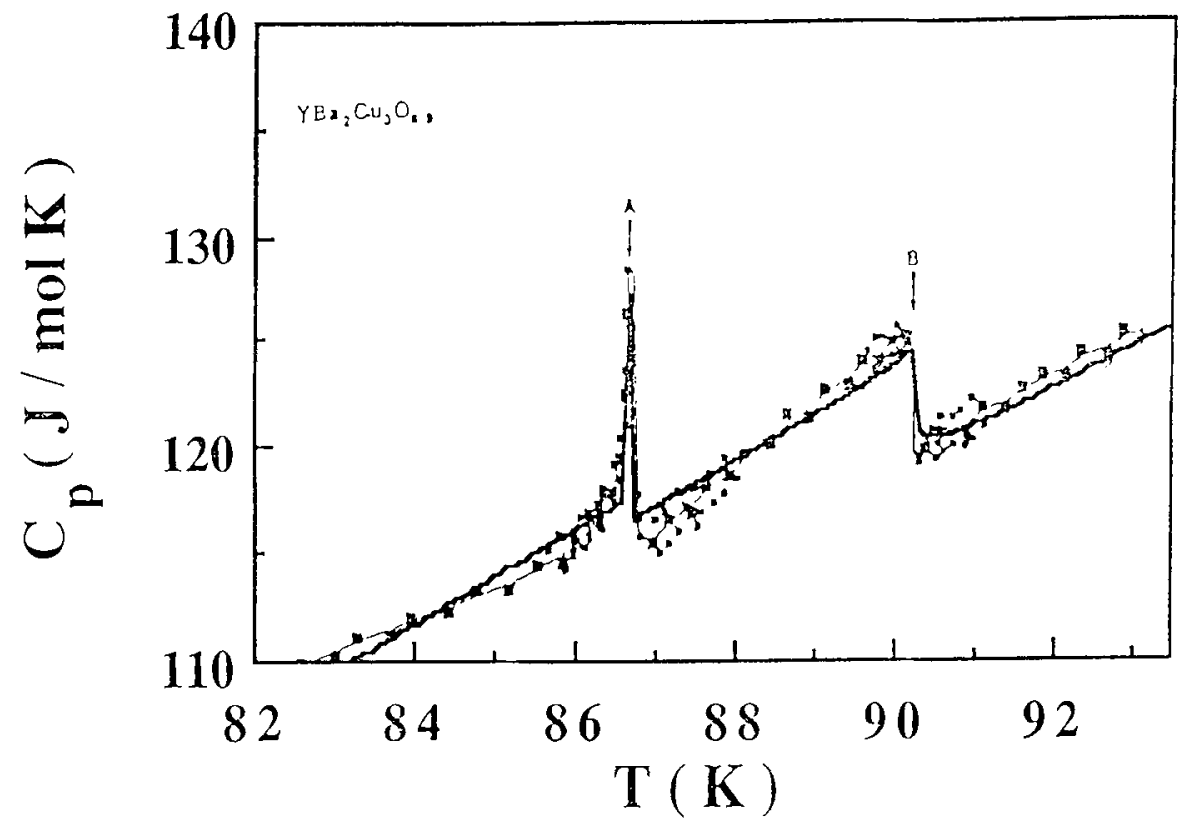

Figure 1. Specific heat as a function of temperature for $\mathrm{YBa}_{2} \mathrm{Cu}_{3} \mathrm{O}_{7-\gamma}$. Solid curve from two-order parameter GL theory including Gaussian fluctuations (Choy et al 1989) and square dots data from Butera (1988). 


\section{Acknowledgement}

I am grateful to Tuck Choy and Hong-Xing He for collaboration.

\section{References}

Abrikosov A A, Budzin A I, Kulic M L and Kuptsov D A 1988 Int. J. Mod. Phys. B1 1045

Butera R 1988 Phys. Rev. B37 5909

Choy T C, Das M P and He H-X 1989 Phase transitions 201

Das M P 1989 in Studies in high temperature superconductors (ed.) A V Narlikar (New York: Nova Science Publication) Vol. 3, p. 267

Ebner C and Stroud D 1988 Calculation of fluctuation contributions to thermodynamic properties of single crystal $\mathrm{YBa}_{2} \mathrm{Cu}_{3} \mathrm{O}_{7-s}$ (Preprint)

Fischer H E, Watson S K and Cahill D C 1988 Commun. Cond. Matter Phys. 1465

Fisher R A, Gordon J E and Phillips N E 1988 J. Supercond. 1231

Horn P, Keane D T, Held G A, Jordan-Sweet J L, Kaiser D L, Holtzberg F and Rice T M 1987 Phys. Rev. Lett. 592772

Inderhees S E, Salamon M B, Goldenfeld N, Rice J P, Pazol B G and Ginsberg D M 1988 Phys. Rev. Lett. 601178,2445

Ma S K 1976 Modern theory of critical phenomena (Mass: Benjamin)

Muzikar P 1988 Phys. Rev. Lett. 61479

Sobyanin A A and Stratonnikov A A 1988 Physica C153-155 1681

Volovik G E 1988 JETP Lett. 4841

Volovik G E and Gorkov L P 1985 Sov. Phys. JETP 61843 